\title{
DEPREDADORES DE MURCIÉLAGOS EN LA CUEVA DE LOS LAGUITOS, CHIAPAS, MÉXICO
}

\author{
MATÍAS MARTÍNEZ-CORONEL, XAZMÍN MORALES-MEDINA Y CAROLINA \\ MÜDESPACHER-ZIEHL.
}

\author{
Laboratorio de Citogenética Animal, Departamento de Biología, Universidad Autónoma \\ Metropolitana-Iztapalapa. Av. San Rafael Attixco 186, Col. Purísima, Iztapalapa, D. F. \\ correoelectrónico:marti17@hotmail.com
}

\begin{abstract}
Resumen: La cueva de "Los Laguitos", localizada cerca de Tuxtla Gutierrez,Chiapas, es por sus condiciones ambientales esta clasificada como una cueva de calor y es habitada por nueve especies de murciélagos, que mantienen una población de más de 100000 individuos en cualquier época del año. La cueva fue visitada 225 veces durante 14 años, tiempo durante el cual registramos la presencia de seis vertebrados silvestres y uno doméstico, considerados depredadores de murciélagos. Los depredadores que fueron observados siempre fueronTrimorphodon biscutatus y Glaucidium brasilianum, seguidos de Didelphis marsupialis, Felis catus, Boa constrictor, Urocyon cinereoargenteus y Geococcyx velox. De las nueves especies de murciélagos que habitan la cueva, solamente Mormoops megallophylla, Pteronotus davyi, P. parnellii, Artibeus jamaicensis, Leptonycteris yerbabuenae y Natalus mexicanus fueron depredadas, mientras que Balantiopteryx plicata, P. personatus y Glossophaga soricina no lo fueron. De los siete depredadores registrados en la cueva, el gato doméstico puede ser el más dañino para esta comunidad.
\end{abstract}

Palabras clave: depredación, murciélagos, serpientes, aves, mamíferos.

\begin{abstract}
Los Laguitos" is a hot cave located near of Tuxtla Gutiérrez, Chiapas. The cave is used as refugee by nine species of bats, with a population greater than 100000 individuals at any time of year. Seven vertebrate predators that feed on bats were registered in the cave in 225 visits along 14 years. The lyre snake, Trimorphodon biscutatus, and the ferruginous pygmyowl, Glaucidium brasilianum, were encountered all times. The common opossum, Didelphis marsupialis was the next most observed species followed by, the domestic cat (Felis catus), the boa constrictor (Boa constrictor), gray fox (Urocyon cinereoargenteus) and lesser roadrunner (Geococcyx velox). Mormoops megallophylla, Pteronotus davyi, P. parnellii, Artibeus jamaicensis, Leptonycteris yerbabuenae and Natalus mexicanus were the bat species captured by these predators, while Balantiopteryx plicata, P. personatus and Glossophaga soricina don't were detected in the carcasses. From the seven vertebrate predators registered in the cave, we consider the domestic cat as the more dangerous for this community to .
\end{abstract}

Key words: predation, bats, snakes, birds, mammals. 


\section{INTRODUCCIÓN}

Los murciélagos son depredados por una variedad de organismos que van desde arañas y ciempiés gigantes hasta reptiles, aves y mamíferos (Gillette y Kimbourgh, 1970; Molinari et al., 2005). Usamos la palabra depredación para referirnos al tipo de interacción donde el depredador representa al organismo que caza y mata a su presa para consumirla (Begon et al., 2005). Entre los reptiles, las serpientes parecen ser las únicas que depredan murciélagos. En la región neotropical las especies que principalmente capturan murciélagos son colúbridos y boídos y en menor proporción los vipéridos (Esbérard y Vrcibradic, 2007). En México se tiene evidencia de la mazacuata (Boa constrictor) y la falsa nauyaca (Trimorphodon biscutatus) como depredadores de estos organismos (Sánchez-Hernández y Ramírez-Bautista, 1992). Dentro de las aves depredadoras de murciélagos sobresalen las rapaces diurnas, como halcones y cernícalos, y nocturnas como búhos y lechuzas, que los capturan cuando salen de sus refugios o cuando están buscando alimento (Chacón-Madrigal. y Barrantes, 2004; Fenton et al., 1994; Fortuna et al., 2009; Hernández et al., 2007; Twente, 1954). Los mamíferos que más frequentemente capturan murciélagos son el gato doméstico, el gato montés y el tlacuache (Gillette y Kimbourgh, 1970; Wroe y Wroe, 1982). Los gatos y tlacuaches los cazan principalmente en la entrada de las cuevas o bien alrededor de los cuerpos de agua cuando bajan a tomar.

La depredación y las condiciones microclimáticas son considerados los factores más importantes en la selección del refugio por parte de los murciélagos (Kunz, 1982; Tuttle y Stevenson, 1982). De todos los refugios empleados por los murciélagos, las cuevas son los sitios más seguros contra los depredadores y los cambios bruscos del ambiente, razón por la cual estos se congregan en las mismas. Sin embargo, grandes congregaciones de murciélagos representan islas de recursos para depredadores (Esbérard y Vrcibradic, 2007; Gillette y Kimbourgh, 1970; Hammer y Arlettaz, 1998; Kunz, 1982; Molinari et al., 2005; Rodríguez y Reagan, 1984; Thomas, 1974; Twente, 1954; Wroe y Wroe, 1982).

Debido a que existe poca información sobre depredadores de murciélagos en México, el objetivo del presente trabajo es dar a conocer información sobre vertebrados que depredan o potencialmente pueden depredar murciélagos en la cueva de los Laguitos, Chiapas. En especial como depredador en esta cueva, queremos enfatizar la presencia del gato doméstico (Felis catus) y las posibles consecuencias que su presencia puede ocasionar a esta comunidad.

\section{ÁREADE ESTUDIO}

La cueva de Los Laguitos se encuentra ubicada al noroeste de Tuxtla Gutiérrez, Chiapas (16 46' 42" LN; 930' 55" LW), a una altitud de $781 \mathrm{msnm}$. Es una cavidad 
horizontal con una sola entrada y una longitud total mayor a $600 \mathrm{~m}$. Por sus condiciones ambientales se considera una cueva de calor, con valores promedio anuales de $32.3^{\circ}$ de temperatura y $95.4 \%$ de humedad relativa (Martínez-Coronel et al., en prensa). Las cuevas de calor son aquellas donde los valores de temperatura y HR son superiores a los valores promedio anuales del ambiente externo, y son más estables en comparación con otros tipos de cuevas (De la Cruz, 1992; Silva-Taboada, 1979). La vegetación que rodea a la cueva corresponde a una selva baja caducifolia (Miranda, 1998). En su interior coexisten nueve especies de murciélagos: Balantiopteryx plicata, Mormoops megallophylla, Pteronus davyi, P. parnellii, P. personatus, Artibeus jamaicensis, Glossophaga soricina, Leptonycteris yerbabuenae y Natalus mexicanus. La población de murciélagos que existe en la cueva a lo largo del año es siempre mayor a 100000 individuos, no obstante de las variaciones numéricas que experimentan algunas especies como L. yerbabuenae, M. megallophylla, P. davyi, P. parnellii y N. mexicanus (Martínez-Coronel et al., 1996; en prensa).

\section{MÉTODOS}

Como parte de otros estudios, la cueva fue visitada durante 225 días entre octubre de 1996 y julio de 2009, tiempo durante el cual fueron cubiertos todos los meses del año. La población de murciélagos fue estimada través de conteos directos en el caso de Balantiopteryx plicata, mientras que en las demás especies se hizo por conteos en áreas de superficie conocida y estos valores fueron extrapolados a toda el espacio que en ese momento ocupaba la colonia.

Durante las visitas en el día o antes del oscurecer, hacíamos una exploración visual en la entrada de la cueva para detectar la presencia de depredadores potenciales de murciélagos, el contacto visual iniciaba a una distancia de $30 \mathrm{~m}$, aún protegidos por la vegetación. Durante la inspección nocturna de la entrada siempre utilizamos luz de lámparas de cacería. Una vez que estábamos en la entrada de la cueva, la inspección fue dirigida a las paredes, techo y piso, donde buscábamos huellas, restos de murciélagos o serpientes. En la parte interna de la cueva, la presencia de los depredadores fue detectada con la ayuda de la luz de las lámparas. En 151 visitas las observaciones iniciaron media hora antes de la puesta del sol y hasta seis horas después; en las 74 visitas restantes realizamos observaciones cada tres horas hasta completar un ciclo de $24 \mathrm{hrs}$. Cada vez que observamos un depredador potencial, determinamos la especie, la hora de observación e inspeccionamos si estaba cazando o alimentándose de algún murciélago. En ciertos casos fue posible determinar a través de los restos o por fotografía la especie del murciélago capturado. 


\section{RESULTADOS}

Durante los 225 visitas a la cueva detectamos la presencia de seis vertebrados silvestres y un vertebrado doméstico (Cuadro 1). Por su frecuencia sobresalen la falsa nauyaca (Trimorphodon biscutatus) y el búho enano o tecolote bajeño (Glaucidium brasilianum), que fueron observados en todas las visitas. La actividad de ambos depredadores ocurre al anochecer, justo cuando los murciélagos emergen de la cueva. En caso de la falsa nauyaca llegamos a contar un máximo de cinco individuos en un mismo día, y en una ocasión observamos que un individuo de aproximadamente 150 $\mathrm{cm}$ de longitud consumió cinco murciélagos en un lapso de dos horas. Estas serpientes se suspenden del techo o se posan sobre las paredes de la entrada y llegan a penetrar hasta 15 metros de profundidad y en ninguna ocasión detectamos que rebasaran éste límite. De todas las veces que observamos a las serpientes, en 31 ocasiones capturaron murciélagos: 16 individuos correspondieron a L. yerbabuenae, 10 a N. mexicanus, 4 a M. megalophylla, 3 a $P$. davyi y 2 a $P$. parnellii. Todas las presas fueron consumidas, excepto cuando un individuo cayó al piso y regurgitó cinco L. yerbabuenae. El comportamiento que mostró la serpiente una vez que atrapaba al murciélago, era de tolerancia a nuestra presencia cuando nos acercamos a determinar la especie de murciélago (Figura 1).

Al igual que la falsa nauyaca, el búho fue observado en los 225 días que visitamos la cueva. La mayoría de las veces fue un ejemplar, excepto en dos días de enero del 2009 que detectamos a dos individuos en el mismo árbol. Éste animal se posaba al atardecer en los árboles que rodean la entrada de la cueva y los cazaba cuando al anochecer. Debido a la oscuridad y a que una vez que capturaba a su presa se alejaba con ella, desconocemos que murciélagos fueron depredados por esta ave.

El tlacuache (Didelphis marsupialis) fue el tercer animal más observado en la cueva, y mostró actividad tanto en el día como en la noche. En noviembre de 1998 encontramos a una madre que llegó a vivir con tres crías a la cueva, y en noviembre del 2001 detectamos a otra madre con cuatro crías. En ambos años los animales fueron vistos tanto día como de noche y durante varios días, alimentarse de neonatos de $L$. yerbabuenae. En junio del 2004 observamos a dos adultos depredando neonatos de $N$. mexicanus en el interior de la cueva.

Los depredadores silvestres con menos avistamientos fueron la mazacuata (Boa constrictor), el correcaminos (Geococcyx velox) y la zorra gris (Urocyon cinereoargenteus). En el caso de la primera, observamos un individuo en agosto del 2004, julio del 2005 y mayo del 2008, y que usó como sitio de caza una cornisa a cuatro metros del piso y a tres de profundidad respecto a la entrada. El correcaminos fue observado salir de la cueva durante el día en noviembre de 1997, mientras que la zorra fue observada saliendo una noche en octubre de 1998. 


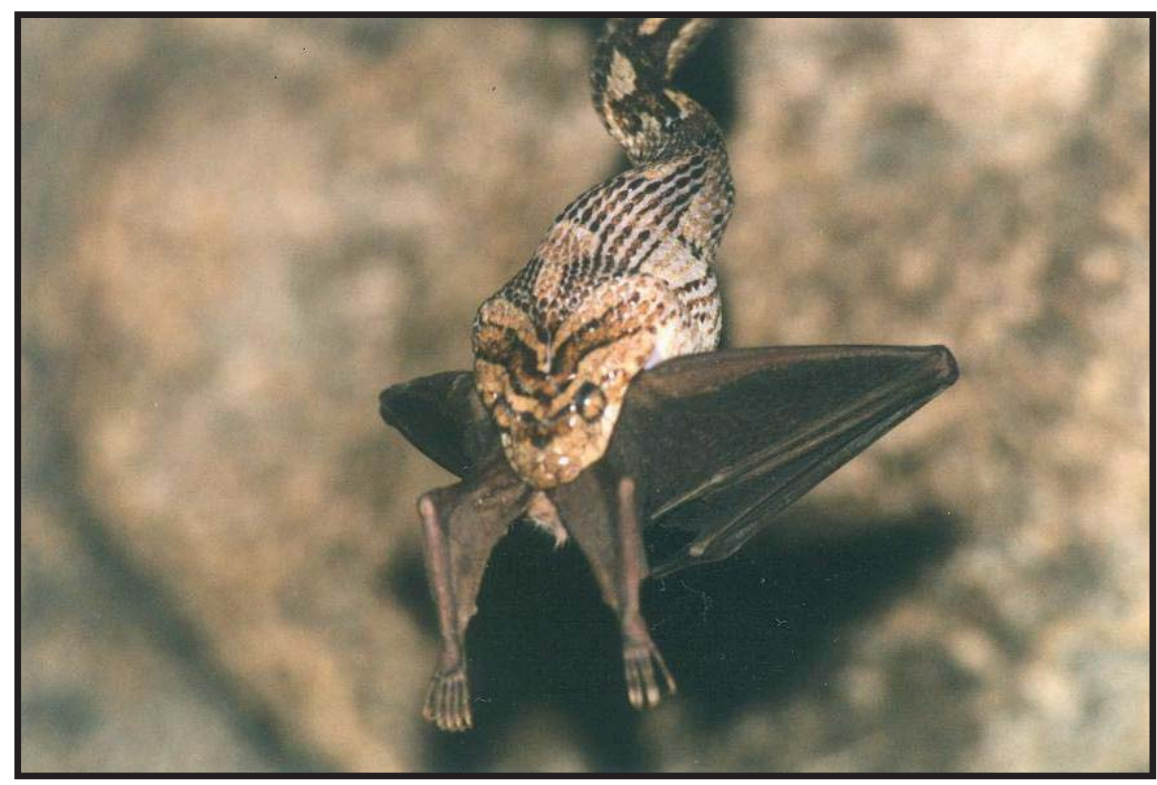

Figura 1. Ejemplar de Trimorphodon biscutatus alimentándose de un individuo de Leptonycteris yerbabuenae en la cueva de "Los Laguitos", Chiapas.

Cuadro 1. Vertebrados depredadores de murciélagos observados en la cueva de "Los Laguitos", Chiapas. El número total de visitas a la cueva fue de 225. En cada depredador se indica el número de veces que fue observado y el porcentaje que esto representa (entre paréntesis).

Grupo Vertebrado Especie Días observados

\begin{tabular}{llrr}
\hline Reptiles & Boaconstrictor & 3 & \\
& Trimorphodon biscutatus & 225 & $(100 \%)$ \\
& & & \\
Gves & Geococcyx velox & $(100 \%)$ \\
& & 1 & \\
& Didelphis marsupialis & 36 \\
Mamíferos & Urocyon cinereoargenteus & 1 \\
& Feliscatus & 4
\end{tabular}


El gato doméstico (Felis catus) fue detectado por primera vez en abril del 2004, cuando observamos a dos individuos salir de la cueva a medio día. El 17 de agosto del 2004 encontramos en la entrada de la cueva restos de 158 murciélagos (Cuadro 2) agrupados discretamente, hecho que llamo nuestra atención porque eran demasiados animales y a todos ellos les faltaba el cuerpo y la cabeza. Ese día recogimos todos los restos y dejamos limpio el piso de la entrada. El día 20 de agosto regresamos a la cueva y observamos a cinco metros de profundidad como dos gatos atrapaban murciélagos al vuelo, y una vez sometido el animal solo consumían el cuerpo y la cabeza, dejando los restos en un mismo sitio. Debido a que detectaron nuestra presencia, estos animales se internaron a la cueva, donde los observamos atrapar neonatos de $N$. mexicanus. Esa noche levantamos restos de 62 murciélagos, que suponemos fueron consumidos entre el día 18 y el 20 de agosto. Con base en lo observado concluimos que los gatos eran responsables del amontonamiento de alas levantadas el día 17 de agosto. Las especies de murciélagos más depredadas fueron $M$. megallophylla, P. davyi, P. parnellii, A.jamaicensis, L. yerbabuenae y N. mexicanus.

Cuadro 2. Especies de murciélagos consumidos por gatos domésticos en la cueva de "Los Laguitos", Chiapas, en agosto del 2004.

\begin{tabular}{lccc}
\hline Especie & 17 agosto & 20 agosto & Total \\
& & & \\
\hline Mormoops megallophylla & 55 & 0 & 55 \\
Pteronotus davyi & 4 & 7 & 11 \\
Pteronotus parnellii & 0 & 4 & 4 \\
Artibeus jamaicensis & 1 & 15 & 16 \\
Leptonycteris yerbabuenae & 0 & 2 & 2 \\
Natalus mexicanus & 98 & 34 & 132 \\
\hline
\end{tabular}




\section{DISCUSIÓN}

De los siete vertebrados observados en la cueva de "Los Laguitos", el búho, el tlacuache, el gato doméstico y la falsa nauyaca, depredaron sobre diferentes especies de murciélagos, tanto de neonatos como de adultos. Los neonatos capturados fueron de las especies L. yerbabuenae y $N$. mexicanus, especies que hemos observado dejan a los críos en sitios tan bajos como $70 \mathrm{~cm}$ del piso, mientras salen a forrajear por la noche. En cambio durante el día siempre los cargan; no obstante llegamos a observar al gato doméstico y al tlacuache llevar críos de estas especies en el hocico, por lo que suponemos que la madre fue sorprendida por el depredador mientras descansaban. En cambio los murciélagos adultos siempre fueron capturados en la entrada de la cueva durante el vuelo de emergencia. Este comportamiento es el más frecuentemente observado tanto en aves como en serpientes en otras cuevas (Fenton et al., 1992; Herreid, 1972).

No todas las especies de murciélagos presentes en la cueva fueron identificadas como alimento de los depredadores, sino únicamente las que tienen poblaciones (mayores a 5000 individuos) a lo largo de todo el año o al menos durante la época reproductiva: $M$. megallophylla, $P$. davyi, $P$. parnellii, A. jamaicensis, $L$. yerbabuenae y $N$. mexicanus. Las especies con poblaciones pequeñas como $B$. plicata (menor a 15 individuos), Glossophaga soricina (alrededor de 500 individuos) y $P$. personatus (alrededor de 4000 individuos durante la reproducción) no fueron detectadas (Martínez-Coronel et al., en prensa). Esto es sin duda consecuencia de un fenómeno probabilístico, ya que por tener poblaciones más pequeñas los individuos de estas especies ven diluida su probabilidad a ser capturados, más no significa que no sean depredados (Begon et al., 2005).

En relación con los vertebrados detectados en la cueva pero que no atraparon murciélagos, suponemos que sí llegan a ella es porque van en busca de estos organismos. Por ejemplo, la literatura registra que la boa se alimenta de $N$. mexicanus en Jalisco, A.jamaicensis en Colombia y Noctilio albiventris en Brazil (Esbérard y Vrcibradic, 2007; Thomas, 1974; Villa y López-Forment, 1966). En cuanto a la zorra gris y al correcaminos tropical, ambos organismos incluyen en su dieta a pequeños vertebrados (Guerrero et al., 2002), y si entraron a la cueva cuando los neonatos de $L$. yerbabuenae eran abundantes, entonces es posible que los hayan consumido.

En la dinámica de una comunidad natural las consecuencias poblacionales de la interacción depredador-presa están reguladas (Begon et al., 2005; Vandermeer y Goldberg, 2003); sin embargo, la presencia de un animal exótico como el gato doméstico puede tener un impacto negativo severo. Esto es, los depredadores silvestres cazan por regla un número de presas de acuerdo a sus necesidades energéticas, a la vez que sus poblaciones se encuentran de alguna manera regulada por la disponibilidad de alimento (Begon et al., 2005). Los gatos domésticos, no obstante que se les alimente 
como mascota, buscan y cazan por naturaleza organismos silvestres para satisfacer sus instintos, llegando en ocasiones a matar un número mayor de presas de las que pueden consumir (Christian, 1975; MacDonald et al., 1984). Aunado a esto, los murciélagos son organismos con un potencial reproductivo bajo, ya que la mayoría de las especies solo se reproducen una vez al año y por norma tienen una cría. En consecuencia, si una comunidad como la que alberga la cueva de "Los Laguitos" se ve amenazada por depredadores tan dañinos como los gatos domésticos, es posible que se encuentre en riesgo de verse alterada de manera irreversible.

Los gatos son especialmente dañinos para la fauna silvestre, en especial de pequeños vertebrados de las zonas rurales o en los límites de las zonas urbanas, como resulta ser el caso de la cueva de Los Laguitos. Una ventaja que tienen los gatos sobre los depredadores silvestre es que se trata de organismos muy adaptables que pueden vivir en cualquier sitio (Apps, 1986). El efecto de la presencia de este depredador es más dramático en las islas, debido a que en algunas de ellas pueden ser los únicos depredadores y por lo tanto los responsables de la extinción de algunas especies (Aguirre-Muñoz et al., 2008; Vázquez-Domínguez et al., 2004).

Las cuevas de calor, como lo es la cueva de "Los Laguitos" son especiales por sus condiciones ambientales, no son ambientes comunes y los organismos que las habitan tienen adaptaciones o requerimientos únicos para soportar la elevada temperatura y humedad atmosférica (De la Cruz, 1992; Silva-Taboada, 1979). Asimismo, es una cueva con una alta diversidad de especies de murciélagos, seis de las cuales están clasificadas como cavernícolas obligados (Arita, 1993; Martínez-Coronel et al., en prensa). Por lo tanto consideramos que es necesario protegerla.

\section{AGRADECIMIENTOS}

A los dos revisores anónimos, cuyas críticas nos permitieron exponer las ideas de manera más clara. Agustín Torres Jiménez, Alma X. Hernández, Edmundo García Hernández, Humberto Posadas Rivera y Rogelio Bautista Trejo nos apoyaron en el trabajo de campo. La UAM-Iztapalapa aportó la mayor parte del apoyo económico para recabar esta información, a través de los diferentes proyectos que se han realizado bajo la dirección de la M. en C. Carolina Müdespacher. Marcelina Blas y familia nos han brindado su casa y amistad todo el tiempo que han durado nuestros proyectos en Tuxtla Gutiérrez.

\section{LITERATURA CITADA}

Aguirre-Muñoz, A., D.A. Croll, C.J. Donlan, R.W. Henry, III, M.A. Hermosillo, G.R. Howald, B.S. Keitt, L. Luna-Mendoza, M. Rodríguez-Malagón, L.M. Salas-Flores, A. Samaniego-Herrera, J.A. Sánchez-Pacheco, J. Sheppard, B.R. Tershy, J. Toro-Benito, S. 
Wolf y B. Wood. 2008. High-impact conservation: Invasive mammal eradications from the islands of western México. Ambio, 37:101-107.

Apps, P.J. 1986. Home ranges of feral cats on Dassen Island. Journal of Mammalogy, 67:199200.

Arita, H.T. 1993. Conservation biology of the cave bats of Mexico. Journal of Mammalogy, 74:693-702.

Begon, M., C.A. Townsend y J.L. Harper. 2005. Ecology: From individuals to ecosystems. 4a. Ed. Wiley-Blackwell, New York.

Chacón-Madrigal, E. y G. Barrantes. 2004. Blue-crowned motmot (Momotus momota) predation on a long-tongued bat (Glossophaginae). The Wilson Bulletin, 116:108-110.

Christian, D.P. 1975. Vulnerability of meadow voles, Microtus pennsylvanicus, to predation by domestic cats. American Midland Naturalist, 93:498-502.

de la Cruz, J. 1992. Bioecología de la cuevas de calor. Mundos Subterráneos, 3:7-21.

Esbérard, C.E.L. y D. Vrcibradic. 2007. Snakes preying on bats: new records from Brazil and a review of recorded cases in the Neotropical Region. Revista Brasileira de Zoología, $24: 848-853$

Fenton, M.B., I.L. Rautenbach, S.E. Smith, C.M. Swanepoels, J. Grosell y J. Van Jaarsveld. 1994. Raptors and bats: threats and opportunities. Animal Behavior, 48:9-18.

Fortuna, M.A., A.G. Popa-Lisseanu, C. Ibáñez y J. Bascompte. 2009. The roosting spatial network of a bird-predator bat. Ecology, 90:934-944.

Gillette, D.D., y J.D. Kimbourgh. 1970. Chiropteran mortality. Pp. 262-281, en: About bats (B.H. Slaughter y D.W. Walton, eds.). Dallas Southern Methodist University Press, Dallas.

Guerrero, S., M.H. Badii, S.S. Zalapa y A.E. Flores. 2002. Dieta y nicho de alimentación del coyote, zorra gris, mapache y jaguarundi en un bosque tropical caducifolio de la costa sur del estado de Jalisco, México. Acta Zoológica Mexicana (n.s.). 86:119-137.

Hammer, M. y R. Arlettaz. 1998. A case of snake predation upon bats in northern Morocco: some implications for designing bat grilles. Journal of Zoology, London, 245:211-212.

Hernández, D.L., J.J. Mell y M.D. Eaton. 2007. Aerial predation of a bat by an American crow. The Wilson Journal of Ornithology, 119:763-764.

Herreid, C.F., II. 1972. Snakes as predators of bats. Herpetologica, 17:271-272.

Kunz, T.H. 1982. Roosting ecology of bats. Pp. 1-55, en: Ecology of bats. (T.H. Kunz, ed.). Plenum Press, New York.

MacDonald, M.L., Q.R. Rogers y J.G. Morris. 1984. Nutrition of the domestic cat, a mammalian carnivore. Annual Review of Nutrition, 4:521-562.

Martínez-Coronel, M., M. Pérez-Gutiérrez y J.R. Albores. 1996. Los murciélagos de la cueva de Los Laguitos: su importancia biológica y social. Revista ICACH, nueva época 2:10-18.

Martínez-Coronel, M., E. García, C. Müdespacher-Zihel y A. Torres. en prensa. El microclima de la cueva de "Los Laguitos" y su relación con los murciélagos. Mundos Subterráneos.

Miranda, F. 1998. La vegetación de Chiapas. Tercera Edición, Gobierno del Estado de Chiapas, Tuxtla Gutierrez.

Molinari, J., E. Gutiérrez, A.A. de Ascenção, J.M. Nassar, A. Arends y R.J. Márquez. 2005. Predation by giant centipedes, Scolopendra gigantea, on three species of bats in a Venezuelan cave. Caribbean Journal of Science, 41:340-346. 
Rodríguez, G.A. y D.P. Reagan. 1984. Bat predation by the Puerto Rican boa, Epicrates inornatus. Copeia, 1984:219-220.

Sánchez-Hernández, C. y A. Ramírez-Bautista. 1992. Life history notes. Trimorphodon biscutatus. Prey. Herpetological Review, 23:121.

Silva-Taboada, G. 1979. Los murciélagos de Cuba. Editorial Academia, La Habana, Cuba.

Thomas, M.E. 1974. Bats as a food source for Boa constrictor. Journal of Herpetology, 8:188.

Tuttle, M.D. y Stevenson, D. 1982. Growth and survival of bats. Pp. 105-150, en: Ecology of bats. (T.H. Kunz, ed.). Plenum Press, New York.

Twente, J.W., Jr. 1954. Predation on bats by hawks and owls. The Wilson Bulletin, 66:135136.

Vandermeer, J.H. y D.E. Goldberg. 2003. Population ecology: First principles. Princeton University Press, New Jersey.

Vázquez-Domínguez, E., G. Ceballos y J. Cruzado. 2004. Extirpation of an insular subspecies by a single introduced cat: the case of the endemic deer mouse Peromyscus guardia on Estanque Island, Mexico. Oryx, 38:347-350.

Villa, B. y W. López-Forment. 1966. Cinco casos de depredación de pequeños vertebrados en murciélagos de México. Anales del Instituto de Biología, UNAM, 37:187-193.

Wroe, D.M. y S. Wroe. 1982. Observation of bobcat predation on bats. Journal of Mammalogy, 63:682-683. 